\title{
Position of retinal blood vessels correlates with retinal nerve fibre layer thickness profiles as measured with GDx VCC and ECC
}

\author{
Hemma Resch, Barbara Brela, Christa Resch-Wolfslehner, Clemens Vass
}

Department of Ophthalmology, Medical University of Vienna, Austria

\section{Correspondence to}

Clemens Vass, Department of Ophthalmology and Optometry, Medical University of Vienna, General Hospital, Währinger Gürtel 18-20, A-1090 Vienna, Austria; clemens.vass@ meduniwien.ac.at

Accepted 3 July 2010 Published Online First 30 August 2010

\begin{abstract}
Aim To evaluate the correlation of the retinal blood vessel position and the retinal nerve fibre layer (RNFL) thickness profile.

Methods RNFL thickness of 81 healthy subjects was measured using scanning laser polarimetry (SLP). To quantify the retinal blood vessel position, the angle (superior and inferior) between a horizontal line and a line from the optic disc centre to the intersection of the most temporal major retinal blood vessel and the outer margin of the measurement ellipse was measured on the SLP printout.
\end{abstract}

Results A negative correlation was found between both the superior and inferior angle and the superotemporal and inferotemporal RNFL thickness, and a positive correlation between both angles and the superonasal and inferonasal RFNL thickness. The steepest slope of the regression line was located in the superotemporal and inferotemporal regions $\left(-0.7\right.$ to $\left.-1.0 \mu \mathrm{m} /{ }^{\circ}\right)$. Using this slope, the difference in RNFL thickness for the interquartile range of the superior angle was $13 \mu \mathrm{m}$. Conclusion RNFL thickness profiles correlate with the location of the main temporal superior and inferior blood vessels. The application of a normative database, taking into account the position of major blood vessels, might improve the diagnostic power of RNFL measurement.

\section{INTRODUCTION}

The GDx (Carl Zeiss Meditec, Dublin, Califonia, USA) is a scanning laser polarimeter that assesses retinal nerve fibre layer (RNFL) thickness in the peripapillary retina and discriminates well between normal subjects and patients with glaucoma. ${ }^{1-4}$

In healthy eyes, the peripapillary RNFL thickness modulation pattern around the optic disc, also referred to as a temporal-superiornasal-inferior-temporal (TSNIT) graph, was shown to follow a double-hump pattern, with thicker RNFL superiorly and inferiorly and thinner RNFL temporally and nasally. ${ }^{5}$ Considerable interindividual variability in RNFL thickness values among normal subjects as measured with optical coherence tomography (OCT) ${ }^{6}$ and some overlap in thickness values between normal subjects and patients with glaucoma have been reported in several studies conducted by scanning laser polarimetry (SLP). ${ }^{2-4} 78$

Various diseases, most notably glaucoma, result in the death of retinal ganglion cells (RGCs) and the degeneration of their axons. The loss of RGC axons in turn leads to a thinning of the RNFL. One method for assessing pathological RNFL appearance is by comparing the RNFL with normative values, derived from healthy subjects. These normative values will be more specific when normal physiological differences are taken into account.

Only recently, Hood et al have demonstrated qualitatively that the local maxima in the RNFL profile of OCT and GDx are associated with blood vessels. ${ }^{9} 10$

Statistical analyses concerning the impact of retinal blood vessel position on the total circumpapillary TSNIT profile of RNFL have not yet been published. Understanding the sources of the intersubject variability is central to the understanding and improvement of RNFL thickness measurements as a test for glaucomatous damage. It was the aim of the present study to examine the influence of the retinal blood vessel position on the RNFL thickness profile around the optic disc as measured with GDx VCC (variable corneal compensation) and ECC (enhanced corneal compensation).

\section{SUBJECTS AND METHODS Subjects}

The study protocol was approved by the Ethics Committee of the Medical University of Vienna and followed the guidelines of Good Clinical Practice and the Declaration of Helsinki. A total of 83 subjects of both sexes aged between 19 and 71 years (mean 27.27 years, \pm 9.5 ) were included.

\section{Inclusion and exclusion criteria}

Inclusion criteria were normal ophthalmic findings, especially normal appearance of the optic disc, normal visual fields and intraocular pressure (IOP), and lack of significant retinal disorder. An abnormal visual field was defined as a glaucoma hemifield test outside normal limits and/or a corrected pattern standard deviation with $\mathrm{p}<0.05 .^{11}$ A normal IOP was defined as $\leq 21 \mathrm{~mm} \mathrm{Hg}$. Any of the following excluded a subject from participation in the trial: evidence of any eye disease except refractive error, history of ocular trauma or intraocular surgery within the last 6 months, ocular inflammation or infection within the last 3 months, astigmatism more than +2.0 dioptres and ametropia of more than \pm 5.0 dioptres.

\section{Experimental paradigm}

Initially a prestudy screening was carried out, where the medical and ocular history was taken. A complete ophthalmological examination was performed, including fundoscopy, visual acuity, 
measurement of IOP by Goldmann applanation tonometry and standard automated perimetry (SAP).

Subjects eligible for participation in the study according to the inclusion/exclusion criteria were included. One eye was selected randomly for the GDx VCC and ECC measurements.

The study was performed at the Department of Ophthalmology, Allgemeines Krankenhaus, Vienna.

\section{Methods}

Automated visual field testing was performed with the Humphrey field analyser II (program 30-2). Visual field eligibility criteria were $<33 \%$ false-positive responses, $<33 \%$ false-negative responses and $<33 \%$ fixation losses.

SLP GDx VCC was performed using a commercial GDx VCC system Version 5.5.0 (NDB Version 1.05.00, Carl Zeiss Meditec, Dublin, California, USA). Additionally we used the GDx ECC method, which provides individualised corneal compensation with enhanced SLP measurement sensitivity. Details of the set-up are described elsewhere. ${ }^{12} 13$ Briefly, SLP assesses RNFL thickness in the peripapillary retina by measuring the RNFL birefringence with a near-infrared diode laser. With GDx ECC software, the corneal polarisation compensator is automatically adjusted so that the combined retardation magnitude from the cornea and the compensating retarder is $\sim 55 \mathrm{~nm}$ with a vertical slow axis of polarisation. This adjustment bias serves to boost the signal to overcome low sensitivity that can make retardation measurements susceptible to optical and electronic noise. After image acquisition, the bias is subtracted to yield the RNFL retardation values.

In our study, each subject with pupils undilated had scans on the same day performed by an experienced operator. The spherical equivalent refractive error was tested subjectively and entered into the software to allow the GDx VCC and ECC to focus on the retina. All selected images were of high quality (quality scan score of $\geq 8$ ) with a centred optic disc, were well focused and illuminated throughout the image, and were without motion artefacts. A fixed concentric measurement band with 27 pixels $(\sim 2.4 \mathrm{~mm})$ inner diameter and 35 pixels $(\sim 3.2 \mathrm{~mm})$ outer diameter was centred on the optic disc, after which the measurements of peripapillary retardation were conducted. Areas of blood vessels are a source of noise and are therefore automatically excluded for analysis by the GDx software. ${ }^{4}$ Retardation was converted to an estimate of RNFL thickness by the software. The GDx data were exported to a personal computer for data analysis.

To examine the retinal blood vessel position we measured the angle between a line through the centre of the optic disc and the most temporal major retinal blood vessel at a particular peripapillary location at 35 pixel distance from the optic disc centre (= outer margin of the measurement band) and between a horizontal line through the optic disc centre. This was performed separately on the GDx printout for the upper and the lower retinal blood vessels using a setsquare. The respective values are referred to as superior angle and inferior angle.

All analyses were done for GDx VCC and ECC. The parameters investigated in this study were RNFL thickness values of 64 sectors ('plots') around the optic disc on the measurement band, and the parameters of the GDx standard printout: TSNIT average, superior average, inferior average, ellipse standard deviation (TSNIT SD), as well as nerve fibre indicator (NFI) and typical scan score (TSS).

\section{Statistical methods}

We calculated linear regression analyses with the superior angle and the inferior angle as independent variables and the RNFL thickness values of 64 sectors of the TSNIT graph as dependent variables. To depict the slopes of the regression lines of the 64 sectors according to the formula $(\mathrm{y}=\mathrm{Kx}+\mathrm{d})$ and their significances, we plotted a modified TSNIT graph using $\mathrm{K}$ values and the upper and lower limits of the $95 \% \mathrm{CI}$ of the $\mathrm{K}$ values. Statistical significance of the slope was reached when the $95 \%$ CI did not include zero. While this analysis yields the clinically relevant information about the effect size (= slope) it is not identical to an analysis of the strength of the correlation. We thus additionally checked the significance of Pearson $\mathrm{R}$ to test the strength of correlation of the superior or inferior angle with each of those sectors.

To test the linearity of regression we additionally performed a stepwise multiple linear regression analysis using the inferior angle and its square and the superior angle and its square. Statistical analyses were done with the SPSS software package (SPSS, Chicago, Illinois, USA) release No. 16.0.2.

\section{RESULTS}

Fourty-four female and 37 male subjects were included in the study. Subjects' baseline characteristics, IOP and visual field mean deviation $(\mathrm{MD})$ are given in table 1 . We were only able to obtain a sufficient measurement quality of 81 out of 83 patients. Hence all data are from 81 subjects.

There was a significant correlation between the superior angle and the inferior angle $(\mathrm{r}=0.344, \mathrm{p}=0.002)$. The refractive error correlated significantly with the superior angle $(r=0.29, p=0.01)$ but not with the inferior angle $(r=0.18, p=0.112)$.

TSS VCC and ECC, superior and inferior angle mean values are summarised in table 2. The correlations of GDx VCC and ECC parameters with the superior angle and the inferior angle are shown in table 3 . For these parameters the main finding was a significant negative correlation between the superior angle and the superior average of RNFL in GDx VCC and GDx ECC.

The slopes of the regression lines of the superior and the inferior angle with the RNFL thickness values of the 64 sectors around the optic disc are displayed in modified TSNIT graphs (figures 1-4). These figures clearly demonstrate a statistically significant negative correlation between the superior angle and the temporal superior and temporal inferior RNFL thickness values, and a (less pronounced) positive correlation between the superior angle and the nasal superior and nasal inferior RFNL thickness values, for both GDx VCC and ECC (figures 1 and 2). The same correlation was found between RNFL thickness values and the inferior angle (figures 3 and 4).

The steepest slope of the regression line was located in the superotemporal and inferotemporal regions $\left(-0.7\right.$ to $\left.-1.0 \mu \mathrm{m} /{ }^{\circ}\right)$. Using this slope, the difference in RNFL thickness for the IOR of the superior angle is $13 \mu \mathrm{m}$. For the $10-90 \%$ range of superior angle $\left(55-89^{\circ}\right)$ this difference amounts to $34 \mu \mathrm{m}$. The maximum $\mathrm{R}$ values were found superotemporally for the superior angle and inferotemporally for the inferior angle $(-0.497$ to -0.627$)$.

Table 1 Subjects' baseline characteristics, IOP and visual field MD

\begin{tabular}{ll}
\hline Age (years) & $27.3 \pm 9.5^{*}$ \\
Sex (female/male) & $44 / 37$ \\
Refractive error (dioptres) & $-0.23 \pm 1.55^{*}$ \\
IOP (mm Hg) & $14.8 \pm 2.4^{*}$ \\
MD (dB) & $-0.13 \pm 0.59^{*}$ \\
\hline
\end{tabular}

*Results are presented as means $\pm \mathrm{SD},(\mathrm{n}=81)$.

$\mathrm{dB}$, decibel; IOP, intraocular pressure; MD, mean deviation. 
Table 2 Mean values of TSS VCC and ECC, superior and inferior angle

\begin{tabular}{lcc}
\hline & Mean values \pm SD & IOR \\
\hline TSS (VCC) & $95.05 \pm 11.39$ & $95.5-100.0$ \\
TSS (ECC) & $98.65 \pm 4.67$ & $100.0-100.0$ \\
Superior angle & $72.1 \pm 11.08$ & $65.0-78.5$ \\
Inferior angle & $77.06 \pm 9.96$ & $71.0-85.0$ \\
\hline
\end{tabular}

ECC, enhanced corneal compensation; TSS, typical scan score; VCC, variable corneal compensation.

\section{DISCUSSION}

The RNFL thickness profiles measured with OCT vary across individuals, even when refractive error and scan quality are taken into consideration. ${ }^{6}$ This variation in RNFL profiles is large, relative to that found owing to measurement error, ${ }^{14-16}$ age $^{17} 18$ or the size of the optic disc. ${ }^{17} 19$ Reference values for RNFL thickness of different ages, ethnic groups and refractive stages are needed to define normality of RNFL thickness.

In the present study we could find a correspondence between the location of retinal blood vessels and the RNFL thickness around the optic disc (TSNIT graph) as measured with SLP. We investigated whether the angle of specific retinal blood vessels - the most temporal major retinal blood vessels superiorly and inferiorly-diverging from the optic nerve head has an impact on the RNFL thickness around the TSNIT graph. We report that smaller superior or inferior angles (oblique retinal vessel course) were associated with thicker RNFL in the superotemporal and inferotemporal regions, but thinner RNFL in the superonasal and inferonasal regions. A more oblique course of the most temporal major retinal blood vessels is thus associated with a more oblique location of the arcuate bundles of retinal nerve fibres.

We are the first to demonstrate a statistical correlation of retinal blood vessel position with RNFL distribution around the optic disc using SLP. This is in correspondence with the results of the original article of Hood et al, ${ }^{10}$ where a correlation was reported between the position of large retinal vessels and the position of RNFL thickness maxima as measured with GDx and OCT. However, this analysis was qualitative not quantitative.

Why should there be an association of retinal blood vessels and the thicker branches of the RNFL? It has been established for several organs and also for the retina ${ }^{20} 21$ that axon cues offer
Table 3 Correlation of GDx VCC and ECC parameters with the superior angle and the inferior angle

\begin{tabular}{|c|c|c|c|c|c|}
\hline & & \multicolumn{2}{|c|}{ Superior angle } & \multicolumn{2}{|c|}{ Inferior angle } \\
\hline & & $\mathbf{r}$ & Significance & $r$ & Significance \\
\hline \multirow[t]{6}{*}{ GDx ECC } & NFI & 0.287 & 0.052 & 0.114 & 0.396 \\
\hline & TSNIT & -0.066 & 0.779 & -0.038 & 0.859 \\
\hline & TSNIT SD & 0.369 & 0.224 & 0.278 & 0.309 \\
\hline & Average superior & -0.351 & 0.014 & -0.232 & 0.075 \\
\hline & Average inferior & 0.079 & 0.617 & 0.011 & 0.940 \\
\hline & TSS & 0.069 & 0.800 & -0.159 & 0.519 \\
\hline \multirow[t]{6}{*}{ GDx VCC } & NFI & 0.212 & 0.129 & -0.025 & 0.845 \\
\hline & TSNIT & -0.348 & 0.132 & 0.058 & 0.781 \\
\hline & TSNIT SD & 0.452 & 0.068 & 0.132 & 0.558 \\
\hline & Average superior & -0.317 & 0.027 & -0.173 & 0.185 \\
\hline & Average inferior & -0.030 & 0.839 & -0.009 & 0.947 \\
\hline & TSS & 0.204 & 0.060 & -0.122 & 0.213 \\
\hline
\end{tabular}

ECC, enhanced corneal compensation; NFI, nerve fibre indicator; TSNIT,

temporal-superior-nasal-inferior-temporal, average; TSNIT SD, ellipse SD; TSS, typical scan score; VCC, variable corneal compensation.

guidance for sprouting and developing vasculature. Vasculature may even share common guidance signals with axons. ${ }^{22} 23$ Considering these findings one should expect some correlation between the location of the superior temporal and inferior temporal arteries and veins, for example, and the distribution of RGC axons (especially at the localisation of the temporal arcuate nerve fibre bundles). As a consequence, the location of the blood vessels may help to predict the variation in the profiles of RNFL thickness because they mark regions of increased axonal density.

Our findings may be of relevance for the diagnostic accuracy of RNFL measurements. Normal eyes with a straight course of RNFL may be more prone to false-positive RNFL defects at the temporal superior or inferior regions, right beside the edge of the arcuate RNFL bundles. On the other hand in glaucomatous eyes with an oblique RNFL course, a small RNFL bundle defect located temporal superior or inferior might be missed by the GDx. This patient would have started off with high RNFL values in these regions and even after a partial loss of RNFL these values might remain within normal limits. The opposite problem might occur in the nasal superior and nasal inferior sectors. These regions, however, are believed to be of less clinical importance for the diagnosis of glaucoma.
Figure 1 Correlation between the retinal nerve fibre layer (RNFL) as measured with $\mathrm{GDx}$ variable corneal compensation (VCC) and the superior angle. The slopes of the regression lines (K) together with the limits of the $95 \% \mathrm{Cl}$ of the slopes are plotted against the 64 sectors (plots). Statistical significance of the slope is reached when the $95 \% \mathrm{Cl}$ does not include the zero line.

The sections of the TSNIT (temporal-superior-nasalinferior-temporal) graph marked with a bold line symbolise significance of Pearson R.

\section{Correlation of superior angle and RNFL (VCC)}

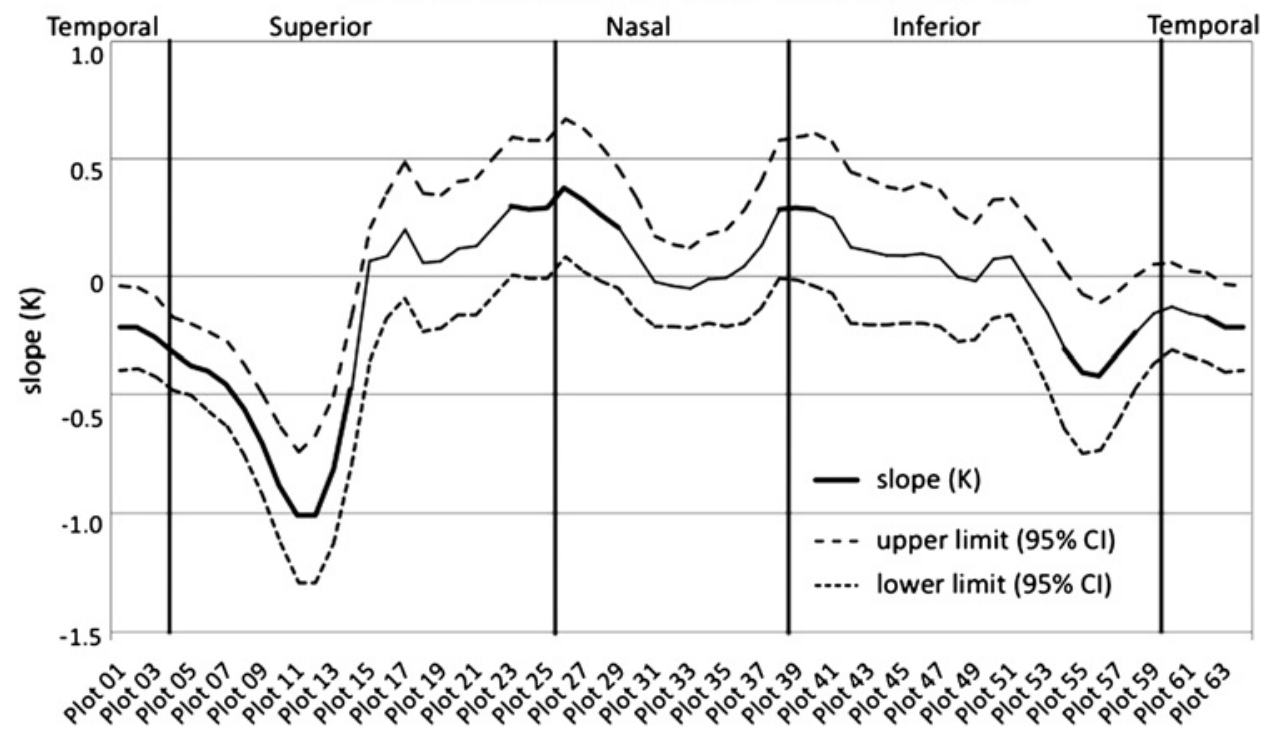


Figure 2 Correlation between the retinal nerve fibre layer (RNFL) as measured with GDx enhanced corneal compensation (ECC) and the superior angle. The slopes of the regression lines (K) together with the limits of the $95 \% \mathrm{Cl}$ of the slopes are plotted against the 64 sectors (plots). Statistical significance of the slope is reached when the $95 \% \mathrm{Cl}$ does not include the zero line. The sections of the TSNIT (temporal-superior-nasalinferior-temporal) graph marked with a bold line symbolise significance of Pearson R.

\section{Correlation of superior angle and RNFL (ECC)}

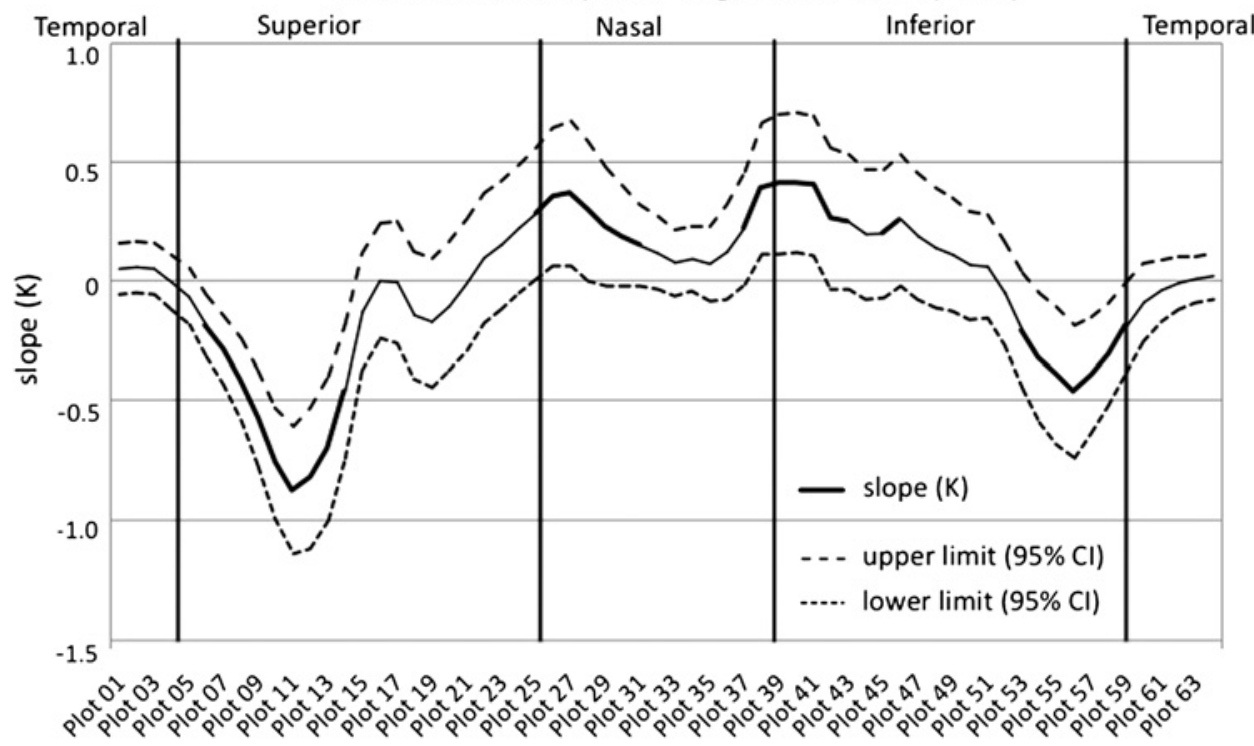

In the clinical situation the problem described above is reduced to some extent by interocular comparison. At least in falsenegative patients with glaucoma, asymmetry in the RNFL distribution is displayed. As a high degree of symmetry is expected in normal eyes, asymmetry is a useful clinical sign of early glaucoma. However, symmetry is sometimes limited in normal eyes and the disease itself may also be symmetric in some patients.

When interpreting the results of this trial a number of intrinsic limitations have to be discussed. We had the aim to test a simple indicator of the retinal vessel distribution for the postulated effect on the RNFL distribution. While we found that even our simple parameter of retinal vessel location had a significant correlation with the thickness of RNFL over onethird to a half of the circumference, we did not test the effect of other retinal vessels. We also did not take into account split bundles that might also influence RNFL thickness. ${ }^{4}$ Split bundles primarily occur in the superior region and only rarely in the inferior region. Our data demonstrate, however, a similar effect of superior temporal and inferior temporal blood vessel location on the RNFL. Split bundles thus do not appear to have greatly influenced our study results. Furthermore, we cannot exclude the possibility that the automatic exclusion of major blood vessel positions from analysis by the GDx software might have influenced our findings.

Another limitation might be the assumption of a linear association between blood vessel position and RNFL thickness. To test a possible non-linear relationship we additionally performed a stepwise multiple regression analysis including a quadratic term of the angle. This analysis demonstrated a non-linear relationship for $25 \%$ of the plots with significant results. However, the effect of non-linearity was small (data not shown).

For clinical use of our findings an automated analysis of the blood vessel position would be necessary. Since the GDx automatically excludes the major blood vessels from the RNFL measurement, the data of blood vessel locations should also be available for analyses similar to ours, although the present software does not offer access to these data.

Finally, the axon density seems to be well coupled to the location of the superior and inferior temporal arteries and veins. A better understanding of the relationship between the distribution of the major blood vessels and GDx RNFL thickness helps us to understand interindividual variation. Reduction of
Figure 3 Correlation between the retinal nerve fibre layer (RNFL) as measured with GDx variable corneal compensation (VCC) and the inferior angle. The slopes of the regression lines (K) together with the limits of the $95 \% \mathrm{Cl}$ of the slopes are plotted against the 64 sectors (plots). Statistical significance of the slope is reached when the $95 \% \mathrm{Cl}$ does not include the zero line. The sections of the TSNIT (temporal-superior-nasalinferior-temporal) graph marked with a bold line symbolise significance of Pearson R.
Correlation of inferior angle and RNFL (VCC)

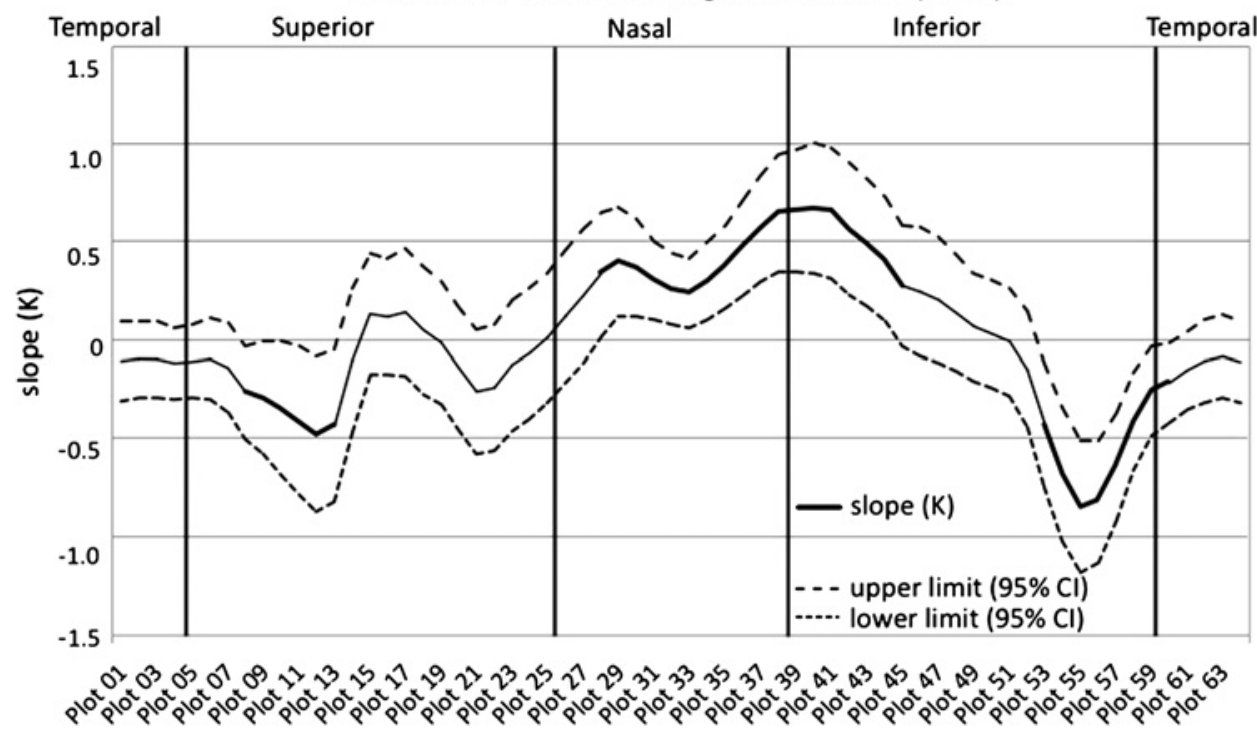


Figure 4 Correlation between the retinal nerve fibre layer (RNFL) as measured with GDx enhanced corneal compensation (ECC) and the inferior angle. The slopes of the regression lines (K) together with the limits of the $95 \% \mathrm{Cl}$ of the slopes are plotted against the 64 sectors (plots). Statistical significance of the slope is reached when the $95 \% \mathrm{Cl}$ does not include the zero line. The sections of the TSNIT (temporal-superior-nasalinferior-temporal) graph marked with a bold line symbolise significance of Pearson R.
Correlation of inferior angle and RNFL (ECC)

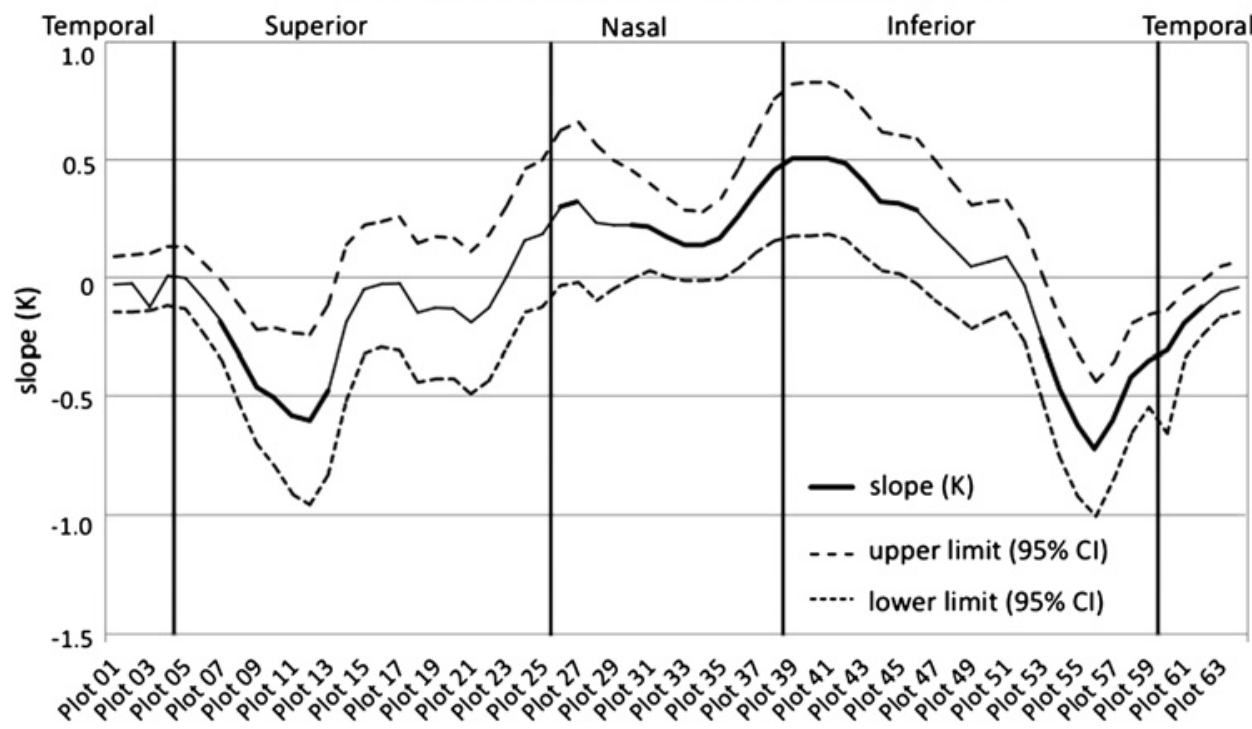

interindividual variation in RNFL profiles may lead to an improvement of specificity and sensitivity of RNFL measurements. The application of a normative database, taking into account the position of major blood vessels, might therefore improve the diagnostic power of RNFL measurement.

\section{Competing interests None.}

Patient consent Obtained.

Ethics approval This study was conducted with the approval of the Ethics committee of the Medical University of Vienna, Austria.

Provenance and peer review Not commissioned; externally peer reviewed.

\section{REFERENCES}

1. Weinreb RN, Shakiba S, Sample PA, et al. Association between quantitative nerve fibre layer measurement and visual field loss in glaucoma. Am J Ophthalmol 1995; 120:732-8.

2. Weinreb RN, Shakiba S, Zangwill L. Scanning laser polarimetry to measure the retinal nerve fibre layer of normal and glaucomatous eyes. Am J Ophthalmol 1995:119:627-36.

3. Choplin NT, Lundy DC, Dreher AW. Differentiating patients with glaucoma from glaucoma suspects and normal subjects by nerve fibre layer assessment with scanning laser polarimetry. Ophthalmology 1998;105:2068-81.

4. Colen TP, Lemij HG. Prevalence of split nerve fibre layer bundles in healthy eyes imaged with scanning laser polarimetry. Ophthalmology 2001;108:151-6.

5. Leung CK, Chan WM, Chong KK, et al. Comparative study of retinal nerve fibre layer measurement by StratusOCT and GDx VCC: I: correlation analysis in glaucoma. Invest Ophthalmol Vis Sci 2005;46:3214-322.

6. Ghadiali $\mathbf{0}$, Hood DC, Lee $\mathrm{C}$, et al. An analysis of normal variations in retinal nerve fibre layer thickness profiles measured with optical coherence tomography. J Glaucoma 2008:17:333-40.

7. Chi $\mathbf{0 M}$, Tomita G, Inazumi K, et al. Evaluation of the effect of ageing on the retinal nerve fibre layer thickness using scanning laser polarimetry. J Glaucoma 1995:4:406-13.

8. Poinoosawmy D, Fontana L, Wu JX, et al. Variation of nerve fibre layer thickness measurements with age and ethnicity by scanning laser polarimetry. $\mathrm{Br} \mathrm{J}$ Ophthalmol 1997:81:350-4.
9. Hood DC, Kardon RH. A framework for comparing structural and functional measures of glaucomatous damage. Prog Retin Eye Res 2007;26:688-710.

10. Hood DC, Fortune B, Arthur SN, et al. Blood vessel contributions to retinal nerve fiber layer thickness profiles measured with optical coherence tomography. J Glaucoma 2008;17:519-28.

11. Keltner JL, Johnson CA, Cello KF, et al. Classification of visual field abnormalities in the ocular hypertension treatment study. Arch Ophthalmol 2003; 121:643-50.

12. Zhou $\mathbf{0}$, Weinreb RN. Individualized compensation of anterior segment birefringence during scanning laser polarimetry. Invest Ophthalmol Vis Sci 2002;43:2221-8.

13. Weinreb RN, Bowd C, Zangwill LM. Glaucoma detection using scanning lase polarimetry with variable corneal polarization compensation. Arch Ophthalmol 2003;121:218-24.

14. Blumenthal EZ, Williams JM, Weinreb RN, et al. Reproducibility of nerve fiber layer thickness measurements by use of optical coherence tomography. Ophthalmology 2000; 107:2278-82.

15. Paunescu LA, Schuman JS, Price LL, et al. Reproducibility of nerve fiber thickness macular thickness, and optical nerve head measurements using StratusOCT. Invest Ophthalmol Vis Sci 2004;45:1716-24.

16. Budenz DL, Chang RT, Huang $X$, et al. Reproducibility of retinal nerve fiber thickness measurements using the stratus OCT in normal and glaucomatous eyes. Invest Ophthalmol Vis Sci 2005;46:2440-3.

17. Bowd C, Zangwill LM, Blumenthal EZ, et al. Imaging of the optic disc and retinal nerve fiber layer: the effects of age, optic disc area, refractive error, and gender. J Opt Soc Am A Opt Image Sci Vis 2002;19:197-207.

18. Hougaard JL, Ostenfeld C, Heijl A, et al. Modelling the normal retinal nerve fibre layer thickness as measured by Stratus optical coherence tomography. Graefes Arch Clin Exp Ophthalmol 2006;244:1607-14.

19. Savini G, Zanini M, Carelli V, et al. Correlation between retinal nerve fibre layer thickness and optic nerve head size: an optical coherence tomography study. Br J Ophthalmol 2005;89:489-92.

20. Stone J, Itin A, Alon T, et al. Development of retinal vasculature is mediated by hypoxia-induced vascular endothelial growth factor (VEGF) expression by neuroglia. J Neurosci 1995;15:4738-47.

21. Stalmans I, $\mathrm{Ng}$ YS, Rohan $\mathrm{R}$, et al. Arteriolar and venular patterning in retinas of mice selectively expressing VEGF isoforms. J Clin Invest 2002;109:327-36.

22. Carmeliet $\mathbf{P}$, Tessier-Lavigne $\mathbf{M}$. Common mechanisms of nerve and blood vessel wiring. Nature 2005;436:193-200.

23. Dorrell MI, Friedlander M. Mechanisms of endothelial cell guidance and vascular patterning in the developing mouse retina. Prog Retin Eye Res 2006;25:277-95. 